\title{
Assisting Tourists on the Move - An Evaluation of Mobile Tourist Guides
}

\author{
Christoph Grün \\ christoph.gruen@ec.tuwien.ac.at \\ Vienna University of Technology, Austria \\ Hannes Werthner \\ hannes.werthner@ec.tuwien.ac.at \\ Vienna University of Technology, Austria
}

\author{
Birgit Pröll \\ bproell@faw.uni-linz.ac.at \\ Johannes Kepler University Linz, Austria \\ Werner Retschitzegger
werner@bioinf.jku.at
Johannes Kepler University Linz, Austria
}

\author{
Wieland Schwinger \\ wieland.schwinger@jku.ac.at \\ Johannes Kepler University, Austria
}

\begin{abstract}
The penetration of high-end mobile devices equipped with GPS and enhanced with multimedia features together with decreasing mobile data prices have resulted in larger usage of mobile services. One of the application domains particularly well-suited for mobile services is tourism, not least since tourists can be assisted especially during the vacation itself. Currently, there is a proliferation of such mobile tourist guides, proposing an unmanageable number of diverse functionalities. To counteract this situation, the contribution of this paper is threefold. First, an evaluation framework is proposed, comprising both, a classification of mobile tourist services and a categorization of their delivery aspects in terms of several orthogonal dimensions. Second, on basis of this framework, four representative mobile tourist guides are evaluated, thereby demonstrating the frameworks' applicability. Third, several lessons learned are discussed, thereby shedding light on the current state of effort in the area of mobile tourist guides.
\end{abstract}

\section{Introduction}

Mobile guides in the tourism domain have come a long way. Since one of the first famous prototypes [2], a large share of research projects (cf. [18]) have been developed so far. Their main goal was primarily to develop working prototypes, to identify previously unknown problems related to certain mobile aspects (e.g., user interfaces or localization means) and to test the prototypes in the field in order to receive first user feedback. Nowadays, the most fundamental problems seem to be eliminated and the sophistication of mobile guides has increased, resulting in the emergence of numerous commercial products (e.g., Mobile Travel Buddy ${ }^{1}$, Mobile Travel Guide ${ }^{2}$, Do Me London ${ }^{3}$ ). The second generation of mobile guides specializes on features such as personalization, recommendation, context-awareness together with new forms of user interaction, collaborative usage and social integration. Their goal is to further push the pace of progress and reduce the market entry's barriers.

In order to shed light on the different approaches in this field, several surveys have already been published [4], [8], [11]. They, however, basically target at the first generation of mobile tourist guides and investigate specific issues such as support for maps or mobility issues, but often do not provide a comprehensive insight into both, the kinds of services which are supported and the more technical aspects of how these services are delivered to the end-user.

In the light of this situation, the contribution of this paper is threefold. First, a comprehensive evaluation framework for second generation mobile tourist guides is provided. This evaluation framework considers both, the services offered to tourists during their on-trip phase and the delivery aspects of these services in

\footnotetext{
${ }^{1}$ http://www.mobiletravelbuddy.com/

2 http://mobiltravelguide.howstuffworks.com/

${ }^{3}$ http://www.do-me.info/
} 
terms of, e.g., customizing information about points of interests (POIs) or assisting tourists in organizing individual tours. Second, the applicability of this evaluation framework is demonstrated by investigating four representative second generation mobile tourist guides. The evaluation is based on in-depth literature reviews as well as partly on hands-on tests of the actual systems. And finally, the findings emerged during this evaluation are reflected by means of several lessons learned, characterizing the current state of the art in the area of mobile tourist guides and pointing the way to further improvements of existing systems.

The paper is structured as follows. Section 2 discusses other surveys conducted so far in the area of mobile tourist guides, whereas Section 3 introduces our evaluation framework consisting of a classification of mobile tourist services and a definition of certain services' design dimensions. This evaluation framework is applied in Section 4 for an in-depth investigation of four mobile tourist guides. The findings and implications for further research are discussed in Section 5, before concluding the paper with a summary in Section 6.

\section{Related Work}

As already mentioned, several surveys on mobile tourist guides have already been published. In the following, we report on this by distinguishing between closely related surveys exploring mobile tourist guides with specific relevance to travelers and more widely related work, aiming at classifying mobile tourist applications. Related work in the area of building up taxonomies of mobile services is mentioned directly in Section 3.

First of all, a survey by Kray \& Baus [11] selected nine mobile guides, each of them offering either some unique features or having large historical influence on the development of mobile guides. The evaluation is based on criteria that are derived from key issues mobile guides have to face, such as adapting the information to the current situation of the user. Apart from giving a brief overview on the provided services, criteria considering adaptivity, user interaction and architecture are considered. The same authors published a more extensive overview of ten mobile guides [4], which are nearly identical to the former, but especially rely on maps in providing their services. It is shown, how maps can be used for mobile guidance or as a major part of the user interface in order to provide users with further services, e.g., information on objects of interests that are represented on the map in form of symbols. Another similar work, done by Eisenhauer et al. [8], reviews the empirical findings of three mobile applications and discusses some design challenges of mobile guides. The empirical findings confirm that a combination of automatic adaptivity based on context information, and manual adaptability, i.e., manual adaptation options for users, is crucial when designing mobile guides.

Although having similar goals, our work tries to approach these goals from a different, more systematic angle, in that our evaluation framework considers several orthogonal dimensions which subsume the aspects discussed in the above mentioned surveys. Beyond that, we evaluate second generation mobile tourist guides, thus considering also criteria, identified by the related surveys as future aspects, such as collaborative usage. And finally, one major focus of our work is on the kinds of services supported from an end-user perspective, representing another orthogonal dimension of our framework.

Less closely related, since dealing with the classification of location-based tourism applications is the work of Stein et al. [20]. The work describes a range of location-based applications and provides a classification according to four criteria, i.e., access technology, location technology, mode of delivery and level of connectedness. The applications have been further classified according to a framework by Giaglis [10] which provides a taxonomy of services, differentiating between emergency, navigation, information, advertising, tracking and billing services. The taxonomy of services is only partly applicable in our context, since it is defined for mobile location services in general whereas our survey focuses on services of the tourism domain. Besides, there might be a crossover between the services of the taxonomy, since, e.g., billing services can be applied to both, information and navigation services. In contrast, our evaluation framework tries to prevent such overlaps between different services by distinguishing several orthogonal dimensions, thus differentiating between the kinds of services supported and the design of these services in terms of e.g., delivery or customization aspects. This allows us to subsume "billing" under the term "transaction" which in turn may be a consumer process of each service (cf. Section 3).

\section{Design of our Evaluation Framework}

The design of our evaluation framework comprises two major issues (cf. Figure 1). First, what mobile services can be identified which are provided for tourists on the move. Second, how those mobile 
services are designed and delivered to tourists in order to generate value proposition and user satisfaction. This issue covers the dimensions service delivery (depicting the services' functionalities), service customization (in terms of adaptation to context) and service initiation (characterizing the mode of delivery).

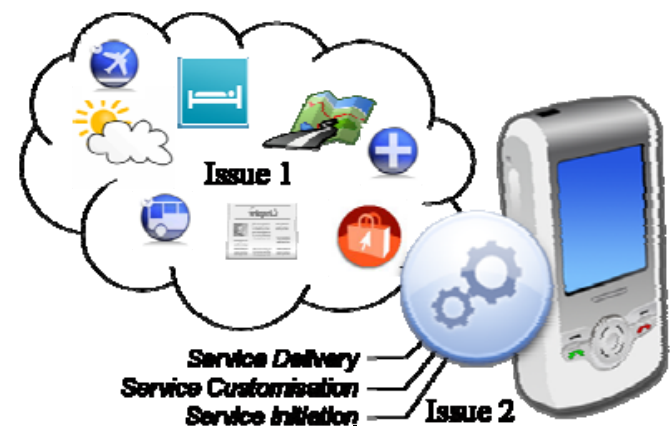

Figure 1. Overall View of our Evaluation Framework

\section{Issue 1 - Classification of Tourist Services}

Mobile services comprise services that can be used independently of temporal and spatial constraints, and that are accessed through a mobile handset [13]. Mobile services in the tourism domain have the task to satisfy information requirements of tourists by providing them with a broad range of travel-related information. To review the mobile services of the different mobile tourist guides, a classification of mobile services is required. Some proposals for a classification can be identified to be useful for our framework. For example, Berger et al. [5] categorize mobile services based on the value chain of tourism which consists of the phases information/booking, transport, accommodation and destination \& information. They further show that each of these phases can be enhanced by location-based technology which provides added-value to travelers, offering localization services (e.g., locate persons or objects), routing services, proximity-based services and information services about travelling conditions.

Rasinger et al. [19] provide a categorization of information requirements posed by tourists in an alpine destination, namely the city of Innsbruck, based on a qualitative-explorative study. Focus group interviews were conducted to refine the findings of the qualitative study, leading to a list of 15 mobile services supporting tourists during their stay. Based on a survey among numerous hotel guests, Murphy et al. [15] examined their information and navigation requirements as a prerequisite for developing location-based services supplementing a typical hotel concierge.

Besides the empirical findings of these field studies, already available classifications of tourism concepts as well as ontologies in the tourism domain have been taken as further sources to build up a reasonable classification of mobile services for our framework. The thesaurus on tourism and leisure activities, a guide to tourism terminology published by the WTO, covers approximately 1800 concepts that are grouped in 20 categories. The harmonise ontology ${ }^{4}$ was defined in an effort to eliminate the different interoperability problems of the European tourism market and ease the distribution of services and data in this market. Basic concepts of this ontology are events, accommodation, attractions as well as food \& drink. There are many other tourism ontologies, such as the GETESS ontology 5 , developed by the Institute of Applied Informatics and Formal Description Methods Karlsruhe (AIFB) or the AUSTO (Australian Sustainable Tourism Ontology) ontology ${ }^{6}$.

The list of mobile tourist services shown in Table 1 is the result of an aggregation of the above mentioned a) service categorization from the field studies and b) tourism concepts of tourism ontologies. The design rationale behind our classification of tourist services was driven by the different information requirements of tourists in the course of their vacation which are induced by varying interests, heterogeneous preknowledge about the destination and continuously changing environmental factors (e.g., weather conditions). The goal of these tourist services is now to respect these requirements appropriately.

Table 1. Mobile information services

\begin{tabular}{|l|l|}
\hline \multicolumn{1}{|c|}{ Services Categories } & \multicolumn{1}{c|}{ Examples } \\
\hline Accommodation & hotels, bed \& breakfast \\
\hline Emergency, Safety, Security & $\begin{array}{l}\text { medical services, urgencies, } \\
\text { pharmacies }\end{array}$ \\
\hline Entertainment & $\begin{array}{l}\text { day trips, excursions, destination- } \\
\text { specific activities, children } \\
\text { activities, cinema/theatre, music, } \\
\text { nightlife, wellbeing }\end{array}$ \\
\hline Gastronomy & restaurants, pubs, clubs \\
\hline Navigation \& Orientation & $\begin{array}{l}\text { maps \& guides, road conditions, } \\
\text { routing service }\end{array}$ \\
\hline News & $\begin{array}{l}\text { political news, business news, } \\
\text { stock quotations }\end{array}$ \\
\hline Practical Information & $\begin{array}{l}\text { tourist information office, car } \\
\text { rentals, cashpoints, currency } \\
\text { money exchange, hairdresser }\end{array}$ \\
\hline Shopping & clothes, gifts, souvenirs \\
\hline Sports & $\begin{array}{l}\text { information on hiking and skiing } \\
\text { tours }\end{array}$ \\
\hline
\end{tabular}

\footnotetext{
4 http://www.harmo-ten.org/

${ }^{5}$ http://www.getess.de/

${ }^{6}$ http://sci.vu.edu.au/ nalin/AUSTOANDIVTRSREPORTv2.19.pdf
} 


\begin{tabular}{|l|l|}
\hline \multicolumn{1}{|c|}{ Services Categories } & \multicolumn{1}{c|}{ Examples } \\
\hline Tourist Attractions & $\begin{array}{l}\text { museums, history, and } \\
\text { architecture, culture, heritage, } \\
\text { sights, churches }\end{array}$ \\
\hline Transport & $\begin{array}{l}\text { flights, port and ferry information, } \\
\text { bus, train, travel assistant }\end{array}$ \\
\hline Weather & weather forecast, temperature \\
\hline
\end{tabular}

The goal of the tourist services is now to react to these requirements by delivering the appropriate information. In order to reach insight into the question how the services can fulfill the user's needs and requirements, in the next section, different design dimensions of mobile tourist services are considered in more detail.

\section{Issue 2 - Design Dimensions of Mobile Services}

The three orthogonal design dimensions of mobile services we distinguish in our work as illustrated in Figure 2, comprise service delivery, service customization and service initiation.

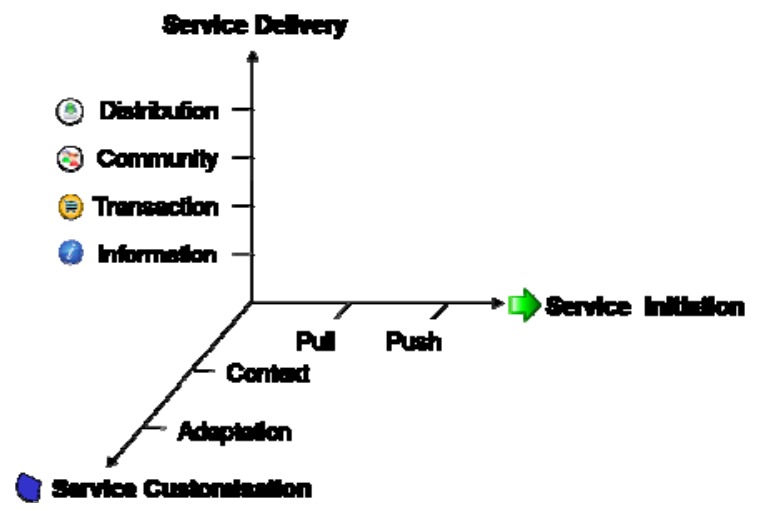

Figure 2. Design dimensions of mobile services

The service delivery dimension identifies the different consumer processes how a user can satisfy her/his requirements. In the simplest form, the user receives information about objects of interests, such as information about restaurants from a gastronomy service. The transaction process allows the user to initiate transactional processes such as reservation, booking or ticketing. The community process enhances the service with features enabling social integration, such as offering possibilities for giving feedback or ratings, tagging or blogging, thus empowering a user to share the tourism experience in a collaborative way. The distribution process enables the user to receive a digital product, such as maps or guides that can be downloaded to the device. In many cases the user has to pay for receiving digital products, either in a subscription-based manner or for a single request.
It has to be noted that this dimension resembles the work of [16] that refines the ICDT (Information, Communication, Distribution, Transaction) model [1] for segmenting the virtual marketspace. In order to better reflect interpersonal communication between community members, we renamed the category "communication" to "community".

The customisation dimension expresses to which extent the information sent to the user is customized to fit the requirements of the respective user. In detail, the level of customisation is characterized by a) the awareness of the current user situation considering context factors such as device, user profile, location or time and b) by the notion of adaptation of services with respect to the context. Several subjects can be affected by the adaptation, comprising content data, user navigation and information presentation. The relevance and quality of services can be further enhanced by integrating different recommenders, leveraging the existing knowledge of the community. Recommenders satisfy the need to know which alternative is best according to the individual purpose, i.e. recommending those tourism products that are most relevant to tourists regarding their current situation.

Concerning the initiation of delivery, services can be classified into pull and push services. Pull services are characterized by a user-triggered search process for retrieving content. The functionality may be improved by including contextual information that may be automatically retrieved. Push services deliver information to the user automatically, i.e., without explicit user request. The information push may be triggered by contextual events (such as changes in the user's location or opening times of attractions). Push services can only be effective, when they are not intrusive and adapt to the situation and requirements of the user.

\section{Evaluation of Mobile Tourist Guides}

\section{Survey Set-up}

A range of mobile tourist guides has already been developed (cf. [3], [7], [12][17]]). Their provided services strongly depend on the type of destination for which they are deployed. For example, compared to a "routing service", a "weather service" may be less important to city travelers than to alpine tourists as for them, certain weather conditions may entail dangerous situations. According to [6], several types of destination can be distinguished, comprising alpine, 
rural or urban destinations. Hence, the offered services may vary according to the type of destination. As a consequence, services can only be evaluated with respect to the destination, in which they are offered. Since most mobile tourist guides have been designed for city tourism, this paper focuses on the urban destination type.

For this survey we pick four mobile tourist guides for urban destinations that have been developed in the last few years, thus belonging to the previously mentioned second generation of mobile guides. We especially focus on systems that are either already used in real situations (etPlanner, mobileStuttgart, Mobile Concierge of Singapore) or have at least been developed and tested in the field (BerlinTainment). Each approach is evaluated in the following according to the evaluation framework that is shown in Figure 3 and described in the next paragraphs.

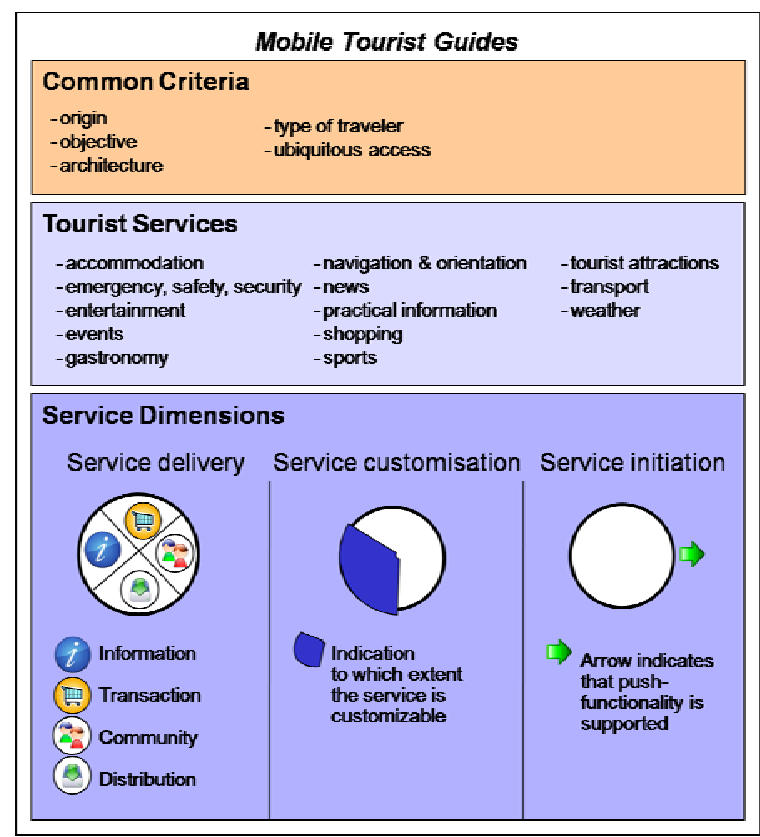

Figure 3. Evaluation Framework

First, criteria that cannot be assigned to merely one tourist service but are rather available to all services and concern the mobile tourist guide in general, are outlined (cf. 1st layer of Figure 3). An overview of the tourist guide is given through the criteria origin, objective as well as architecture. The criterion traveler type refers to the functionality of mobile tourist guides to address the needs of various travelers such as business travelers or tourists traveling alone or in groups. Services should be accessible in a multilingual, multimodal and device-independent manner, thus enabling ubiquitous access. Multilingualism should not be limited to the user interface, but also be applied to the content. Multimodality allows services to be used via textual, graphical, gesture- or speech-based interfaces. Mobile users not only switch between modalities but also between different devices, thereby utilizing different devices such as mobile phones, PDAs and personal computers. Hence, mobile tourist guides should enable users to access information with different end-devices.

Second, the service portfolio of each mobile tourist guide is outlined based on the classification given in the previous section of the paper (cf. 2nd layer of Figure 3). Thereby, a detailed insight for each provided service is given, based on the dimensions service delivery, service customization and service initiation (cf. 3rd layer of Figure 3).

Besides a textual description of each mobile tourist guide, the results of the evaluated mobile tourist guides are presented in a single matrix, thus facilitating comparison and providing an overall view of all evaluated tourist guides. Thereby, the provided services, which are displayed in form of circles, form the intersection points. The respective service delivery types are visualized through appropriate symbols, as far as they are offered to the user. The degree of customization is symbolized through a filled cake slice. For each context factor and each adaptation subject a slice is used. If the whole circle is filled, it indicates that the service is fully customizable, meaning that all context factors and all adaptation subjects are supported. In this case, all six context factors and adaptation subjects must be supported. An arrow next to the circle indicates the support of pushfunctionality for this service.

\section{Overview on Mobile Tourist Guides}

\section{BerlinTainment}

BerlinTainment [21] offers a framework for assisting service providers in developing context-aware entertainment services in large metropolitan areas. It has been developed at the Technical University of Berlin. Despite its more scientific nature it is intended to deploy BerlinTainment as a commercial service for large-scale events. The framework is based on multi agent system technology offering personalization, context-awareness and device independence. BerlinTainment uses a thin client approach. No information is stored on the end-user device, enabling users to switch devices in a seamless way (e.g., using 
the desktop PC for preparation purposes and the mobile phone while being on the move). External data in form of maps and routing information is integrated from the provider map\&guide ${ }^{7}$. BerlinTainment's user interface is available in the languages German and English. BerlinTainment supports multi-modality by offering user interfaces for browser- and voice-based interfaces as well as different end devices. To show BerlinTainment's applicability, different prototypical services in the entertainment domain have been realized in the BerlinTainment demonstrator, providing tourists and residents with information about leisure activities in the metropolitan area of Berlin.

Service Categories. A navigation \& orientation as well as transport service is provided that assist users in finding the way to a specific location either by car or public transport. A map together with the route highlighted and a route description is presented or a public transport itinerary is shown with detailed information on the different means of travel as well as departure times. The event and entertainment services comprise a movie, a music and a stage finder. The gastronomy service is implemented in form of a restaurant finder. These services enable users to initiate a profile-independent search for information concerning restaurants, operas, ballets, theatres or concerts. A tourist attraction service provides information on diverse points of interest such as sightseeing. In addition, information about pharmacies are presented as well as taxi stands, gas stations and ATMs based on a given location. A range of secondary services is provided, comprising office services such as calendar or currency converter. A special kind of information service, namely the "Intelligent Day Planner", allows users to schedule different activities or venues mentioned above, followed by receiving context-aware recommendations based on their individual profiles.

Design Dimensions. The recommendation functionality provides tailored information to users based on their preferences, the location of the venues as well the individual starting times. While contact details in form of telephone number or address are available, no explicit booking or reservation of events is supported by the services. All those services that are integrated in the day-planner service allow users to give feedback in form of ratings. All supported services offer a basic pull functionality. The search for music and stage events, movies and restaurants can be refined by considering different criteria such as

\footnotetext{
${ }^{7}$ http://www.mapandguide.de/
}

location, rating or type of category. In addition, these criteria can be weighted by the user. The notification service enables active information delivery about restaurants, stage events, music events and movies via e-Mail, SMS or voice notification.

Basically, all services make use of device and time context. Whereas device context is integrated in form of CC/PP profiles, the latter is considered dynamically in order to take into account the opening hours of facilities or to select appropriate means of travel according to their time schedule. The navigation \& transport service additionally considers location context. Location information has to be entered in form of street address or district as soon as the user initiates a search or asks the way to a specific location. At start-up, the user can fill out a profile, which is then dynamically and automatically updated by tracking user interactions. This user context is then used by the event service and culture, heritage \& sights service to offer personalized information.

Adaptations are performed by querying suitable content according to user preferences and context and adapting its outcome to different modalities or enddevices. Basically, all three domains are subject of adaptation. The information content is adapted by applying diverse filtering techniques in order to provide personalized information to the user. The structure of the user interface is adapted to the constraints of the device by e.g. reducing images to textual links due to display size restrictions or supported media. Information presentation can be adapted by changing the modality or reducing the size of images.

\section{etPlanner}

etPlanner [14], developed by the competence center $\mathrm{ECCA}^{8}$ in Austria, is a platform for interactive mobile travel guidance for the destination Innsbruck. It is based on a client-server architecture, whereby the application can be accessed either using the browser on the PC or on the mobile phone. Two languages are supported, namely German and English. The tourism content stems mainly from the destination management system Tiscover ${ }^{9}$. A running pilot of the system is available.

\footnotetext{
${ }^{8}$ http://www.etourism-austria.at/

${ }^{9}$ http://www.tiscover.com/
} 


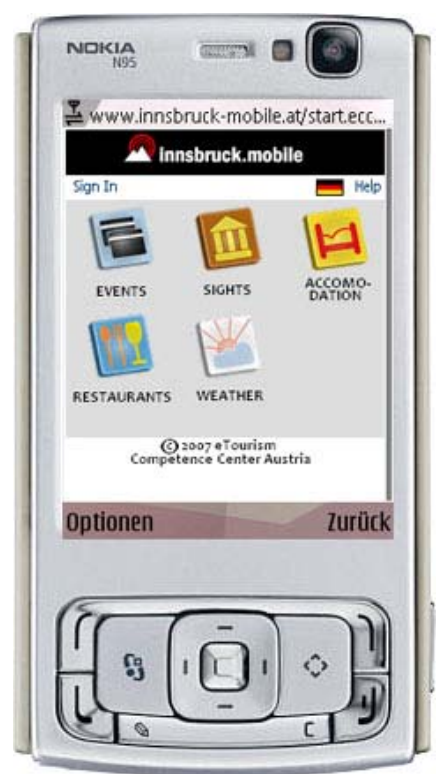

Service Categories.

The start screen provides access to five services, providing information concerning events, tourist attractions, accommodation, gastronomy and weather. All mentioned services provide detailed information to users. As an example, for each event, the event service provides a description, together with date, contact number, price as well as a picture together with a map of the location. Favorite objects of interest can be stored in a watch list in form of a bookmark. The weather service provides a weather forecast for the next three days. Moreover, the system offers the possibility to send the user every morning a "good morning post”, i.e., information about tourist attractions, about current events that take place, or about the current weather conditions either via SMS or via email.

Design Dimensions. Transaction services, such as direct booking of an accommodation, are not provided. Instead, the user can get in contact with the provider by calling the phone number presented at each object of interest. Concerning community services, only rating of the objects of interest is supported by the system. The rating functionality is open for everyone, i.e., users do not have to log in. For the events and tourist attraction services, a dialogue-oriented knowledge-based recommender provides personalized tips to the user based on their personal profile. Basically, all services allow access in a pull-based manner. In addition, the system offers the mechanism to push information to tourists depending on their context. The notification service is based on eventcondition-action rules. External events such as location, weather or time events can trigger rules that may generate push messages that are sent to the user either via e-mail or SMS. Moreover, the user can set the preferred time and date when he wants to receive the push message as well as the mode of delivery (SMS or email).
Table 2. Evaluation results at a glance

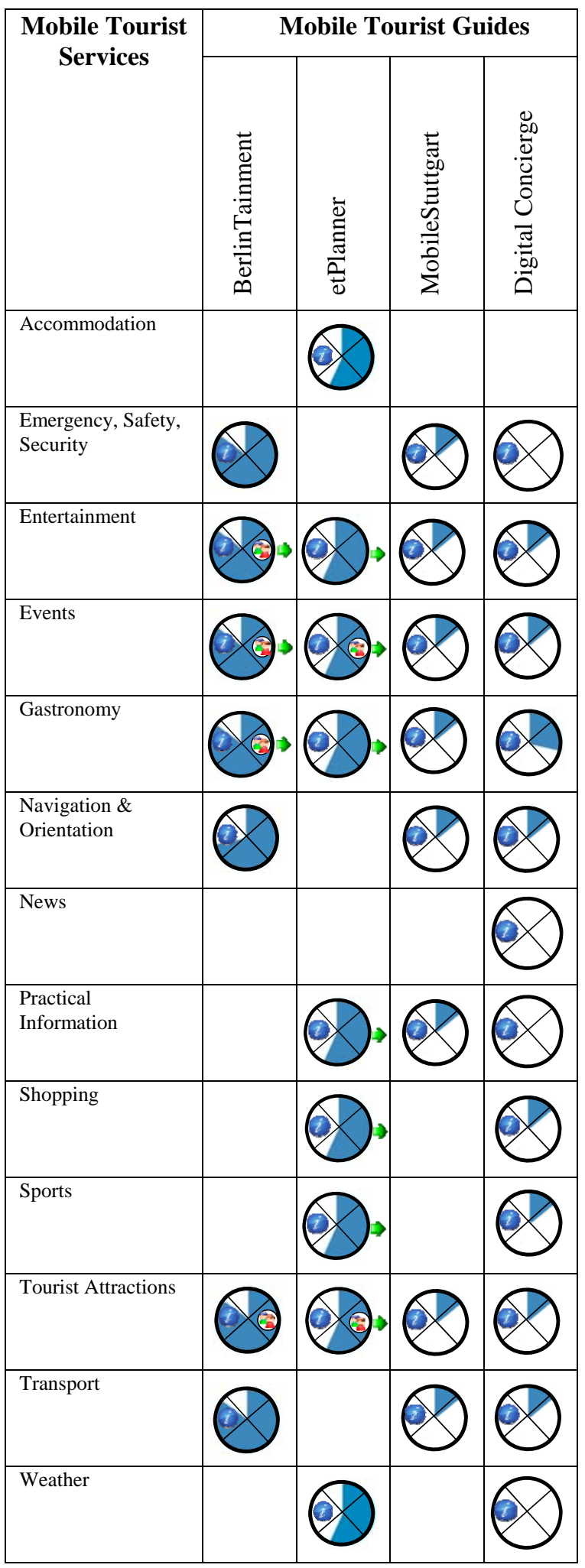




\section{MobileStuttgart}

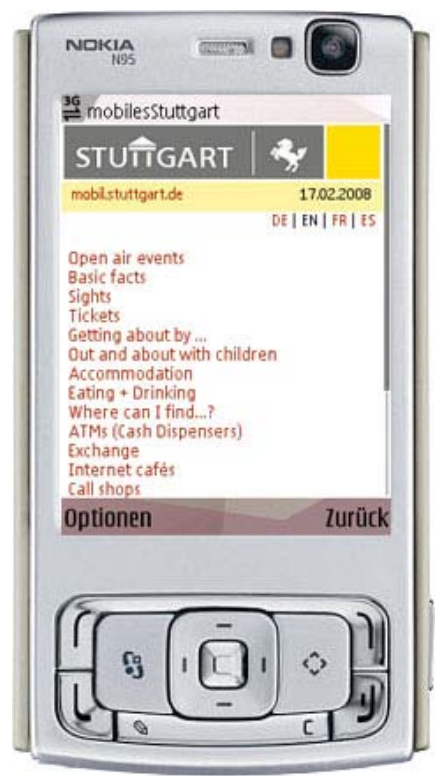

MobileStuttgart [9] is a mobile city guide that provides tourism content about the city of Stuttgart in Germany. The system is implemented based on a client-server architecture, whereby the user interacts with the system by using the browser on the client device. The system was developed on the occasion of the football world cup 2006 in Germany. Its services and content data have been designed and constructed so that they also support citizens as well as tourists. Its user interface is available in four different languages, including German and English.

Service Categories. A full range of services is provided, such as services that provide basic facts about the city as well as information about tourist attractions, gastronomy facilities, events and practical information concerning internet cafes, currency exchange, toilets or emergency services. Whereas the event service delivers dynamical information, the objects of interests, provided by the tourist attraction service are rather static and offer the user information about the location together with a map, contact details and address, public transport information to get there, as well as a basic description and a picture. Basically, all telephone numbers can be directly called when clicked. The transport service not only delivers basic information concerning the chosen mode of transport (e.g., airplane, bus or train) but also provides a comprehensive time schedule. Based on the input of starting and destination address as well as favored time, the transport service lists a set of possible routes, including public transport numbers, time and map of the stops.

Design Dimensions. MobileStuttgart offers a search in order to filter objects of interest that are situated near the user. The user has to enter his/her current address or can choose a prominent landmark from a drop-down menu. The result list is sorted based on the distance. The chosen object from the result list can also be used as the starting point for further searches. Besides the location, device information is considered by querying an integrated device database that comprises more than 1800 devices with more than 8000 profiles that store information concerning resolution or browser. All three subjects of the adaptation are supported. Information objects, mark-up elements and tables, that are not supported by the end device are either removed or transformed, the navigation is adapted and the information presentation is adjusted to the size of the screen and supported graphic formats. All information can only be queried in a pull-based manner.

\section{The Digital Concierge}

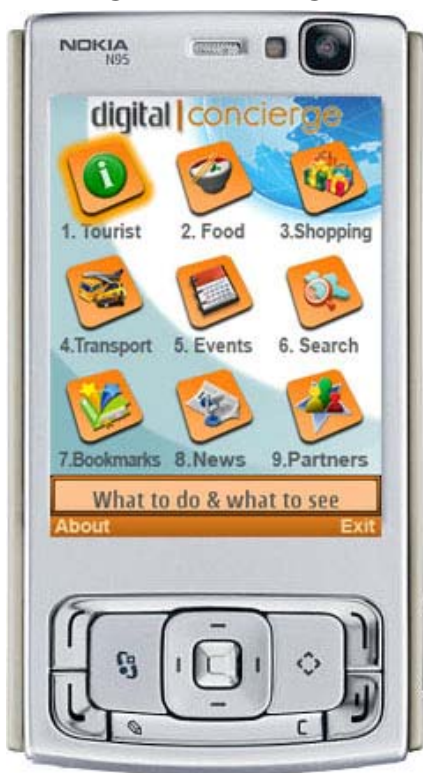

The system “digital concierge"10 aims to enhance the visitor's experience in Singapore by acting as a personal assistant on the mobile phone and providing lots of tourism content such as information on places to visit and dine, current events, currency exchange rates or weather information.

Currently it is running as a pilot test to receive feedback and suggestions for improvement, a full implementation is foreseen in mid 2009. It is being developed by Frontline Technologies and the Cellcity Pte Ltd, and supported by the Infocomm Development Authority of Singapore and Singapore Tourism Board. Since it is a Java based application, it has to be installed on the mobile phone. All Java MIDP 2.0 phones are supported.

Service Categories. The start screen provides access to a range of tourist services, comprising gastronomy, shopping, transport, events, entertainment, tourist attractions and news. Several auxiliary services are provided to the user, such as a currency converter or a service called “traveller's essentials", including emergency numbers, pharmacies, information about the Singapore tourist pass and 20 unique things to do in Singapore. The gastronomy service provides information concerning bars, café, and different kinds

\footnotetext{
10 http://www.digitalconcierge.sg/
} 
of restaurants. The shopping service delivers information about electronics, shoes, shopping malls or souvenirs. The user can get special offers in form of promotions from the shopping or gastronomy service. The transport service informs the user about basic information concerning the airport shuttles, buses, taxis or private cars. Apart from having the possibility to directly call a taxi company, no time schedule or routing functionality is provided. Flight information can be requested by entering a flight number and date. The event service provides information about featured events, events that take place this week as well as the capability to search for events. For an event, basic information is provided, comprising name, date, small description, price and contact address as well as a link to the event's homepage. The news service delivers news about sport, world, business as well as news concerning the region Asia-Pacific. A comprehensive weather service is offered, providing detailed weather information for today or the next days. In addition, the user can view an online weather and satellite map. A watch list provides information to the hotel where the user stays as well as information about key landmarks.

Design Dimensions. All services are implemented based on the pull-approach. Besides navigating through the information by choosing a tourist service from the main screen, a detailed search is provided. It offers the possibility to search places, services and information in yellow pages based on keywords and location. In addition, the location of the results (e.g., hotels) can be viewed on a map. If the mobile device supports GPS, the user can view his/her current position in order not to get lost.

Besides using the location, either chosen from a list or provided automatically through GPS, the user can enter his profile (e.g., preferences concerning type of cuisine) at system start up. In order to provide personalized information, the system exploits location information to filter those results that are situated in the nearer surroundings of the user's current position. The gastronomy service uses the user profile to recommend gastronomy facilities. Since a suitable version that fits to the device in use has to be downloaded and installed, it is ensured that all information is presented in an optimal way.

\section{Lessons Learned}

In this Section, we will briefly summarize the results of our comparison by pointing out the major issues of the approaches surveyed by reporting on lessons learned.
All services more or less provided. The evaluation results show that each service is at least covered by one mobile tourist guide. From the results, one can see that the tourist attractions, events, entertainment and gastronomy services are the most provided services for tourists. Thus, providers of mobile tourist guides should especially focus on these services and provide well implemented, customized versions in order to differentiate themselves from others.

Community, distribution and transaction functionalities widely disregarded. Community aspects are only provided in the form of user feedback and ratings. There are no means to stay in touch with the community while being on the move through provision of micro-blogging services, location-based chats \& messaging as well as ad-hoc coordination between group members. This would enable travelers to share the visits and impressions during their travels in an ad hoc fashion with others. Further, transaction functionalities are not provided by any of the evaluated mobile tourist guides. One reason could be that tourism products are complex goods that are most often consumed in form of bundles. Moreover distribution functionality such as the provision of downloading a city map to the mobile device is not provided by any of those mobile guides.

Potential of customisation not exploited. Most mobile tourist services either provide location-based information such as sorting the search results based on the current position or concentrate on delivering personalized information, such as filtering restaurants based on the user profiles. What is missing is the combined usage of many context factors, e.g., location and user preferences in order to provide more relevant information to the user.

Push-approach still neglected. Pushing relevant information to the users can relieve them from the tedious task of looking it up by themselves. Only etPlanner uses a context-aware push mechanism to provide information that is tailored to the users. This shows that pushing services are still an open research field for the future.

\section{Summary}

This paper presents the state of the art in mobile tourist guides of the second generation, based on a comprehensive evaluation framework. The framework extends past ones by providing both, a classification of services, as well as three design dimension of how those services can be customized for the end user. In this way, the evaluation framework attempts to give a holistic view of mobile tourist services from a value- 
in-use perspective. This survey differs from existing ones since the focus is moved from technical issues and mobile constraints of mobile applications towards an emphasis on the overall value of the services. The evaluation framework helps providers of mobile tourist guides to obtain a holistic view on the services and to develop strategies how they can differentiate their services from others. In addition, the evaluation framework can be used as the basis for customer field studies on how they react to different kinds of services with respect to service delivery, service initiation and service customization.

\section{References}

[1] Anghern A. (1997). The strategic implications of the internet', Proceedings of the Fifth European Conference on Information Systems 1163-1173, Cork Publishing Ltd, Cork (ISBN 1-86076-953-5)

[2] Abowd, G., Atkeson, C., Hong, J., Long, S., Kooper, R., Pinkerton, M (1997). Cyberguide: A mobile contextaware tour guide. ACM Wireless Networks.

[3] Anegg, H., Kunczier, H., Michlmayr, E., Pospischil, G., Umlauft, M. (2002). LoL@: Designing a Location Based UMTS Application. ÖVE-Verbandszeitschrift e\&i, Springer.

[4] Baus, J., Cheverst, K., Kray, Ch. A Survey of Mapbased Mobile Guides, in Liqiu Meng and Alexander Zipf (eds.): "Map-based mobile services - Theories, Methods and Implementations". Springer, Berlin, Heidelberg, New York, (2005).

[5] Berger, S., Lehmann, H., Lehner, F. (2003). Locationbased services in the tourist industry. In Journal of Information Technology \& Tourism. Vol 5 pp. 243-256.

[6] Buhalis, D. (2003). eTourism: Information technology for strategic tourism management. Published by Prentice Hall

[7] Cheverst, K., Davies, N., Mitchell, K., Friday, A., Efstratiou, C. (2000). Developing a contextaware electronic tourist guide: some issues and experiences. In Proceedings of the SIGCHI Conference on Human Factors in Computing Systems (The Hague, The Netherlands, April 01 - 06, 2000). CHI '00. ACM Press.

[8] Eisenhauer, M., Oppermann, R., Schmidt-Belz, B. (2003). Mobile information systems for all. In: Proceedings of the Tenth International Conference on Human-Computer Interaction 2003. pp. 354-358.

[9] Engelbach, W. et al.: Plattformen und Anwendungen für ortsbezogene mobile Dienste: Fallstudien kommerzieller Lösungen für kleine und mittelständische Unternehmen; Erarbeitet im Projekt LOMS "Local Mobile Services"; Unter Mitwirkung der LOMS Partner infoman AG und Siemens Business Services. Stuttgart: Fraunhofer IRB Verlag, 2007, pp. 59-73

[10] Giaglis, G. M., Kourouthanassis, P., and Tsamakos, A. (2003). Towards a classification framework for mobile location services. In Mobile Commerce: Technology, theory, and Applications, B. E. Mennecke and T. J. Strader, Eds. IGI Publishing, Hershey, PA, 67-85.

[11] Kray, C., Baus, J. (2003). A survey of mobile guides. Workshop HCI in mobile guides at Mobile HCI, Italy.

[12] Krösche, J., Baldzer, J., Boll, S. (2004). MobiDENKMobile Multimedia in Monument Conservation. IEEE MultiMedia, 11(2).

[13] Heinonen, K., Pura, M. (2006). Classifying Mobile Services. Presented at Helsinki Mobility Roundtable.

[14] Höpken, W., Fuchs, M. et al. (2006). etPlanner: An IT Framework for Comprehensive and integrative travel guidance, in: ENTER 2006 Proceedings, Information and Communication Technologies in Tourism, Springer.

[15] Murphy, H., Schegg, R. (2006): Information Requirements of Hotel Guests for Location Based Services: Identifying Characteristic Segments. In: Hitz, M., Sigala, M., Murphy, J. (eds.): Information and Communication Technologies in Tourism, Wien, Springer-Verlag, pp. 248-259.

[16] Obrien, P. (2007). An Ontology for Mobile Situation Oriented Systems, Proceedings of the $18^{\text {th }}$ Australasian Conference on Information Systems, Toowoomba.

[17] O'Grady, M.J., O'Hare G.M.P. (2004). Gulliver's Genie: Agency, Mobility \& Adaptivity. Computers \& Graphics, Special Issue on Pervasive Computing and Ambient Intelligence - Mobility, Ubiquity and Wearables Get Together, Vol. 28, No. 4, Elsevier.

[18] Pugh, S., M. Jupp \& F. Collomb (2004). Project POSITION - Supporting the Exploitation of Innovative Tourism Technologies. Information and Communication Technologies in Tourism, Cairo, Springer.

[19] Rasinger, J., Fuchs, M., Höpken, W. (2007) Information Search with mobile Tourist Guides: A Survey of Usage Intention, in: Information Technology and Tourism, 9(3).

[20] Stein, A., Hawking, P., Sharma, P. (2005). A Classification of U-Commerce Location Based Tourism Applications. In AusWeb: The Eleventh Australasian World Wide Web Conference. Ed(s). Andrew Treloar. Southern Cross, Lismore, Qld. 224-232.

[21] Wohltorf, J., Cissee, R., Rieger, A. (2005). BerlinTainment: an agent-based context-aware entertainment planning system, IEEE. On page(s): 102109, Volume: 43, Issue: 6. 